This is an electronic reprint of the original article. This reprint may differ from the original in pagination and typographic detail.

Author(s): Erkkilä, Anna-Leena; Leppänen, Teemu; Ora, Markku; Tuovinen, Tero; Puurtinen, Ari

Title: Hygroexpansivity of anisotropic sheets

Year: $\quad 2015$

Version:

Please cite the original version:

Erkkilä, A.-L., Leppänen, T., Ora, M., Tuovinen, T., \& Puurtinen, A. (2015).

Hygroexpansivity of anisotropic sheets. Nordic Pulp and Paper Research Journal, 30(2), 325-334.

All material supplied via JYX is protected by copyright and other intellectual property rights, and duplication or sale of all or part of any of the repository collections is not permitted, except that material may be duplicated by you for your research use or educational purposes in electronic or print form. You must obtain permission for any other use. Electronic or print copies may not be offered, whether for sale or otherwise to anyone who is not an authorised user. 


\section{Hygroexpansivity of anisotropic sheets}

\section{Anna-Leena Erkkilä, Teemu Leppänen, Markku Ora, Tero Tuovinen and Ari Puurtinen}

KEYWORDS: Hygroexpansivity, Dimensional
$\begin{aligned} & \text { instability, Drying shrinkage, Anisotropy, Fiber } \\ & \text { orientation }\end{aligned}$

SUMMARY: Dimensional instability, more particularly its component hygroexpansivity, may cause problems in process or end-use situations in which paper or board is in contact with water or subject to changes in ambient relative humidity. Misregistration in printing, curl during copying and calender wrinkles are examples of such defects. In this paper, the in-plane hygroexpansivity of oriented laboratory sheets with different pulps and dried both freely and under restraint is studied.

A linear relationship between the drying shrinkage and hygroexpansion coefficient of freely dried laboratory sheets having different fiber orientation anisotropies, was observed. Regardless of both the measurement direction (MD or CD) and the drying options (freely or restraint) all hygroexpansion coefficient values of each pulp type fell quite well on one single power curve as a function of the elastic modulus. Fiber orientation is considered via two different approaches: using fiber orientation anisotropy and using directional variable named as anisotropy index. When the anisotropy index is used, the $\mathrm{MD}$ and $\mathrm{CD}$ hygroexpansivity or the MD and $\mathrm{CD}$ drying shrinkage can be fit on a single curve, while the freely dried and restraint-dried sheets evidently need two different fitting curves. Between the hygroexpansion coefficient and the anisotropy index, a simple power law relationship, with two fitting parameters depending on pulp and drying restraints, is introduced.

ADDRESSES OF THE AUTHORS: Anna-Leena
Erkkilä (anlejoer@gmail.com), Lappeenranta University
of Technology, P.O. Box 20, FI-53851 Lappeenranta,
Finland; Teemu Leppänen (teemu.leppanen@lut.fi) and
Ari Puurtinen (ari.puurtinen@lut.fi), LUT Savo
Sustainable Technologies, Lappeenranta University of
Technology, Varkaus unit, Osmajoentie 75, FI-78210
Varkaus, Finland; Markku Ora (markku.ora@upm.com)
UPM Research Centre, FI-53200 Lappeenranta, Finland
Tero Tuovinen (tero.tuovinen@jyu.fi), University of
Jyväskylä, P.O. Box 35, FI-40014 Jyväskylä, Finland
Corresponding author: Anna-Leena Erkkilä

The term 'dimensional instability' of paper or board refers to dimensional changes resulting from a change in the conditions of ambient air or from moisture exchange in converting processes. Dimensional instability manifests itself as length, width and thickness changes and as out-of-plane deformations. The latter is induced by inhomogeneity in dimensional changes arising from variations in the structure and composition of the paper or board. Dimensional instability causes problems in the paper or board during drying, converting, printing, copying, storage and end-use, referring to such phenomena as curl, cockling, wrinkles, creasing, wavy edges, misregistration, stack lean, shrinkage, surface roughening, grainy edges, deformation of boxes and mechano-sorptive creep.

Depending on changes in the temperature and on the relative humidity of the air, fibers of lignocellulosic materials absorb, adsorb or desorb water. Most of the hygroexpansivity of fibers takes place diametrically; the changes in the length and cross sections are on the order of $1 \%$ to $2 \%$ and $20 \%$ to $50 \%$, respectively, over the range of relative humidity (RH\%) (Page, Tydeman 1962). The lateral hygroexpansivity of the fibers is transferred to the macroscopic dimensional changes of the paper sheet via inter-fiber bonding (Rance 1954; Page, Tydeman 1962; Uesaka 1994; Nanko, Wu 1995). The fiber composition, degree of refining and chemical modifications influence the dimensional stability of paper, while anisotropic fiber orientation generates the anisotropic inplane hygroexpansivity of the sheet (Stamm, Beasley 1961; Page, Tydeman 1962; Fahey, Chilson 1963; Uesaka 1994; Lyne et al. 1996; Li et al. 2009). Hygroexpansivity and its anisotropy are also highly dependent on the hygroscopic, thermal and mechanical history of the sheet (Nordman 1958; Fahey, Chilson 1963; Salmén et al. 1987a; Uesaka et al. 1992; Uesaka, Qi 1994).

In this paper, the in-plane hygroexpansivity of oriented laboratory sheets made from two different pulps and their mixture is studied. The oriented laboratory samples are formed by using a dynamic sheet former and dried either freely or under restraint. Samples from a production machine are used for comparison purposes. The relation of the hygroexpansion coefficient on the elastic modulus, elastic modulus anisotropy, drying shrinkage, fiber orientation anisotropy and anisotropy index is considered. Some of the observed dependencies, together with the experimental stress-strain curve parameters presented in Erkkilä et al. (2013), have been incorporated as a part of the continuum mechanical modeling approach presented in Lipponen et al. (2009) and Erkkilä et al. (2015).

\section{Materials and Methods}

The two pulps used in this study were bleached softwood kraft pulp made from pine (SW) and thermomechanical pulp (TMP). The mixture (MIX) of these contained 71\% TMP and 29\% SW. Drainability (Canadian Standard Freeness (CSF)) determined according to ISO 52672:2001 standard was $592 \mathrm{ml}$ for SW, $57 \mathrm{ml}$ for TMP and $112 \mathrm{ml}$ for MIX pulp. The average fiber lengths were measured on a Kajaani FS300 fiber analyzer. The lengthweighted values were $2.41 \mathrm{~mm}$ for $\mathrm{SW}, 1.46 \mathrm{~mm}$ for TMP and $1.82 \mathrm{~mm}$ for MIX pulp. The production machine (PM) samples taken from middle and edge areas of the cross profile had a fiber composition corresponding to the MIX pulp samples.

The oriented laboratory sheets were formed using a dynamic sheet former. The target basis weight was $65 \mathrm{~g} / \mathrm{m}^{2}$. At the dynamic sheet former, the stock is sprayed against a wire of the rotating drum by the traversing nozzle. The water of the fiber suspension is 
drained away by centrifugal dewatering pressure, forming a wet sheet on the fabric (for a detailed description of the process, see e.g. Norman (1989)). The adjustable velocity difference between the jet and the drum wall allowed for varying degrees of fiber orientation in the sheet. The jet speed varied from $800 \mathrm{~m} / \mathrm{min}$ to $1020 \mathrm{~m} / \mathrm{min}$, and the wire speed from $700 \mathrm{~m} / \mathrm{min}$ to $1200 \mathrm{~m} / \mathrm{min}$ resulting in speed differences of the jet and wire from $-400 \mathrm{~m} / \mathrm{min}$ to $320 \mathrm{~m} / \mathrm{min}$. The sheets were wet-pressed according to ISO5269-1:2005, and then were either air-dried freely on a flat Teflon covered plate or under restraint in standard atmosphere conditions $\left(23^{\circ} \mathrm{C} ; 50 \% \mathrm{RH}\right)$. The elastic modulus (or tensile stiffness) and thickness of single sheet were measured following ISO 1924-2:1994 and ISO 534:2005 standards, respectively.

The procedure for the hygroexpansivity measurements followed the general guidelines stated in ISO 82261:1994 for maximum relative humidity up to $68 \%$. The samples were conditioned under zero load at least $8 \mathrm{~h}$ in RUMED Climatic Test Cabinets Type 4201, with the constant atmosphere in accordance with the DIN 50015. The changes in the MD and CD lengths of the sample were measured using the image analysis-based determination. The round sample with constant diameter was marked with crosshairs at center lines and scanned. During scanning a round metal weight was used to hold the sample in plane with constant pressure. The coordinates of the sample's peripheral were determined via image analysis to obtain the machine direction (MD) and cross machine direction (CD) lengths of round paper samples at $33 \% \mathrm{RH}$ and $66 \% \mathrm{RH}$. According to ISO 8226-1:1994, the hygroexpansive strain $X$ is

$$
X=\frac{l_{66}-l_{33}}{l_{50}} \times 100
$$

where $l$ is the length in the specific RH\% indicated by the subscript. However, the hygroexpansion coefficient $\beta(\% / \%)$, defined as

$$
\beta=\frac{X}{\Delta M}
$$

where $\Delta M(\%)$ is the moisture content change, was used in this study. At least seven parallel samples were measured in each anisotropy level. The same image analysis-based method was also applied in determining the drying shrinkage $S$ of freely dried samples as a percent relative in-plane dimensional change (\%) during drying. Sheet forming and measurements of hygroexpansivity as well as drying shrinkage are described in detail in Hii (2008).

The fiber orientation anisotropy was measured using the method presented in Erkkilä et al. (1998) and Lipponen et al. (2009). The measurement is based on layering the sample by tape ripping and image gradient analysis of the layers. Mass-weighted averages of the MD/CD ratios of the layers are used to describe the fiber orientation anisotropy of the entire thickness. The anisotropy index $\phi$ introduced by Erkkilä et al. (2013) is defined by

$$
\phi_{\gamma}=\sqrt{\frac{1-\xi^{2}}{\xi+\tan ^{2} \gamma / \xi}+\xi}
$$

where $\xi$ is the fiber orientation anisotropy and $\gamma$ is the direction in which the anisotropy index is determined.
For the semi-axes of the orientation distribution, the anisotropy indices can be solved from Eq 3 to be $\phi_{90^{\circ}}=\sqrt{\xi}$ and $\phi_{0^{\circ}}=1 / \sqrt{\xi}$. If the orientation angle coincides with the machine direction, $\phi_{90^{\circ}}$ corresponds to the machine and $\phi_{0^{\circ}}$ to the cross direction anisotropy index values.

The goodness of fit is estimated using the general form of the coefficient of determination defined by the equation

$$
r^{2}=1-\frac{\sum\left(\sigma_{\text {estimated }}-\sigma_{\text {measured }}\right)^{2}}{\sum\left(\sigma_{\text {measured }}-\overline{\sigma_{\text {measured }}}\right)^{2}}
$$

Negative results of the coefficient of determination $r^{2}$ are also possible, indicating that the mean of the measured results would have provided an estimate preferable when compared to the fitting under consideration.

\section{Results and Discussion}

\section{The relationship between elastic modulus and fiber orientation}

Wahlström and Mäkelä (2005) suggest that for restraintdried (rd) and freely dried (fd) samples the following relationships between elastic modulus anisotropy (or tensile stiffness) and fiber orientation anisotropy hold:

$$
\frac{E_{r d, 1}}{E_{r d, 2}}=\xi \text { and } \quad \frac{E_{f d, 1}}{E_{f d, 2}}=2 \xi-1
$$

where $E$ is the elastic modulus (or tensile stiffness) and subscript indices 1 and 2 refer to $\mathrm{MD}$ and $\mathrm{CD}$, respectively. These equations are used for comparing the elastic modulus anisotropy and the fiber orientation anisotropy results of this study (see Fig 1). As can be expected, the PM samples, which are outside the scope of Eq 5, deviate clearly from the diagonal in Fig 1. Otherwise, the sheets produced by the dynamic laboratory sheet former and dried either freely or under restraint follow the diagonal line reasonably well, although some nonlinearity may be observed.

Earlier observations indicate that under equal drying conditions, the geometric mean of MD and CD tensile stiffness remains constant with varying anisotropy levels (Schrier, Verseput 1967; de Ruvo et al. (1976); Htun, Fellers 1982). The relation between tensile stiffness of isotropic sheet and geometric mean value may be written separately for the restraint and the free drying as

$$
E_{\text {iso }}=E_{\text {geom }}=\sqrt{E_{1} E_{2}}
$$

where subscripts iso refers to the isotropic, and subscript geom refers to the geometric mean. The geometric mean of the elastic modulus of the samples studies is presented in Fig 2 as a function of the anisotropy of the elastic modulus $\left(E_{1} / E_{2}\right)$. The geometric mean of the elastic modulus of freely dried samples is roughly equal for all the suspensions studied (SW, TMP and MIX). For the SW samples, restraint during drying increases the elastic modulus by four times, while for the TMP samples, the level is doubled. The geometric means of the front-side and middle samples taken from the cross profile of the production machine differ from each other because of the different $\mathrm{CD}$ restraints prevailing during drying. 


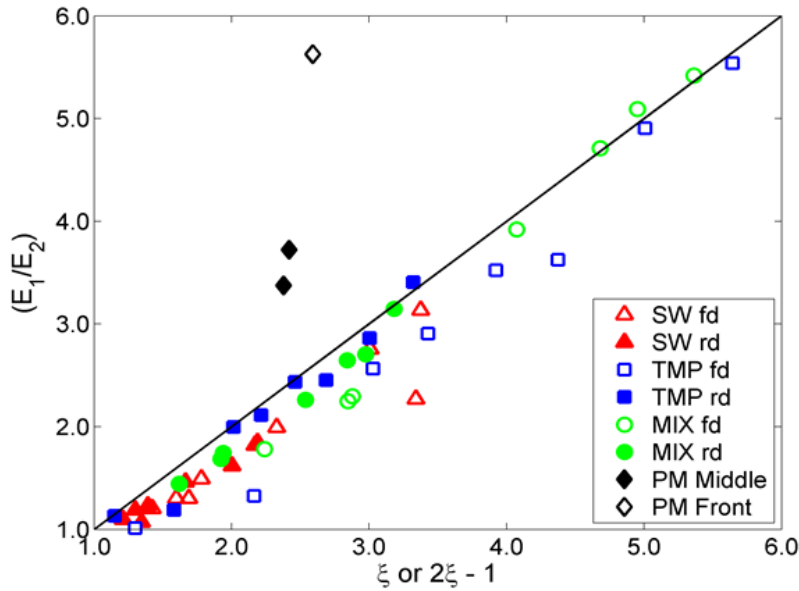

Fig 1 - The elastic modulus anisotropy as a function of fiber orientation anisotropy according to $E q 5$. Eq 5 for restraint drying $(r d)$ is used for production machine (PM) samples.

Table 1 - Geometric mean values of the elastic modulus and coefficient of determination $r^{2}$, when Eq 7 and 8 are applied.

\begin{tabular}{llll}
\hline Pulp and drying & $\boldsymbol{E}_{\text {geom }}(\mathrm{GPa})$ & $\boldsymbol{r}^{\mathbf{2}}(\mathrm{MD})$ & $\boldsymbol{r}^{\mathbf{2}}(\mathrm{CD})$ \\
\hline SW fd & 1.165 & 0.582 & 0.542 \\
SW rd & 4.074 & 0.396 & 0.344 \\
TMP fd & 1.105 & 0.797 & 0.741 \\
TMP rd & 1.959 & 0.881 & 0.887 \\
MIX fd & 1.115 & 0.767 & 0.798 \\
MIX rd & 2.595 & 0.782 & 0.721 \\
\hline
\end{tabular}

In different studies, the parameter $E$ found in $E q 5$ and 6 refers to the tensile stiffness, tensile stiffness index or elastic modulus. If the basis weight and the thickness of comparable samples are equal, these equations are equally accurate, independent of which of the three parameter referents is used. If $E q 5$ and 6 are valid, then the geometric mean of the elastic modulus (or the elastic modulus of the isotropic sheet) and the fiber orientation anisotropy can be used to predict the MD and $\mathrm{CD}$ values of the elastic modulus of an anisotropic sheet, both for free and restraint drying conditions:

$$
\begin{aligned}
& E_{f d}=E_{\text {geom }}(2 \xi-1)^{s} \\
& E_{r d}=E_{\text {geom }} \xi^{s}
\end{aligned}
$$

where $s$ is 0.5 for the direction 1 (MD) and -0.5 for the direction 2 (CD). Fairly good predictability can be observed from Fig 3 (colored lines). The geometric mean values (averaged over different anisotropies) and coefficients of determination $\left(r^{2}\right)$ can be seen from Table 1.

If the orientation angle is zero, such as in the case of the laboratory sheets under consideration, the anisotropy index $\phi(E q 3)$ has the value $\sqrt{\xi}$ for the machine direction and $1 / \sqrt{\xi}$ for the cross direction. Then, by using the anisotropy index, Eq 8 can be simplified to

$$
E_{\text {rd }}=E_{\text {geom }} \phi \text {. }
$$

Eq 9 is applicable to all in-plane directions. The elastic modulus of both the freely and restraint-dried samples as

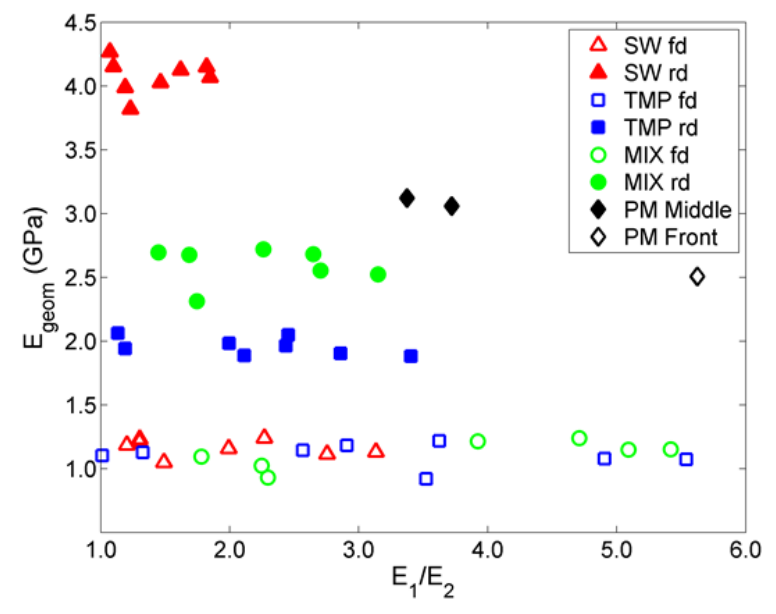

Fig 2 - Geometric mean of the elastic modulus vs. the anisotropy of the elastic modulus for freely dried (fd), restraintdried (rd) and production machine (PM) samples.

Table 2 - Fitted parameters ( $E q$ 10, Fig 4 ) and the coefficient of determination.

\begin{tabular}{llll}
\hline Pulp and drying & $\boldsymbol{k}_{\mathbf{1}}$ (GPa) & $\boldsymbol{q}$ & $\boldsymbol{r}^{\mathbf{2}}$ \\
\hline SW fd & 1.165 & 1.283 & 0.969 \\
SW rd & 4.073 & 0.729 & 0.953 \\
TMP fd & 1.103 & 1.362 & 0.968 \\
TMP rd & 1.964 & 0.935 & 0.980 \\
MIX fd & 1.062 & 1.603 & 0.964 \\
MIX rd & 2.591 & 0.917 & 0.979 \\
\hline
\end{tabular}

Table 3 - Fitted parameters (Eq 11, Fg 3) and the coefficient of determination $\left(r^{2}\right)$.

\begin{tabular}{llllll}
\hline Pulp and & $\boldsymbol{k}_{\mathbf{1}}$ & MD & CD & MD & CD \\
drying & $(G P a)$ & $\boldsymbol{q} \times \boldsymbol{s}$ & $\boldsymbol{q} \times \boldsymbol{s}$ & $\boldsymbol{r}^{\mathbf{2}}$ & $\boldsymbol{r}^{\mathbf{2}}$ \\
\hline SW fd & 1.165 & 0.642 & -0.642 & 0.939 & 0.766 \\
SW rd & 4.073 & 0.365 & -0.365 & 0.905 & 0.750 \\
TMP fd & 1.103 & 0.681 & -0.681 & 0.892 & 0.842 \\
TMP rd & 1.964 & 0.467 & -0.467 & 0.914 & 0.900 \\
MIX fd & 1.062 & 0.801 & -0.801 & 0.856 & 0.369 \\
MIX rd & 2.591 & 0.458 & -0.458 & 0.866 & 0.797 \\
\hline
\end{tabular}

a function of the anisotropy index is presented in Fig 4. Parameters $k_{1}$ and $q$ of the equation

$$
E=k_{1} \phi^{q}
$$

are fitted separately to the results of the freely dried and restraint-dried samples. The fitting parameters are presented in Table 2. The power $q$ for the restraint-dried samples is moderately close to one which is expected because of the fairly good correlations when $E q \quad 8$ is employed. For freely dried samples, the power $q$ is over 1, compensating the term $(2 \xi-1)$ in $E q 7$. The constants $k_{1}$ are virtually equal to the geometric mean values (see Table 1). Eq 10 can be written as a function of anisotropy

$$
E=k_{1} \xi^{q \times s} \approx E_{\text {geom }} \xi^{q \times s}
$$

where $s$ is 0.5 for MD and -0.5 for $\mathrm{CD}$. The fittings of $E q$ 10, but with the change of variable according to $E q$ 11, are plotted in Fig 3 as black lines. The power $q \times s$ and coefficient of determination $r^{2}$ are presented separately for the MD and CD relationships in Table 3. 


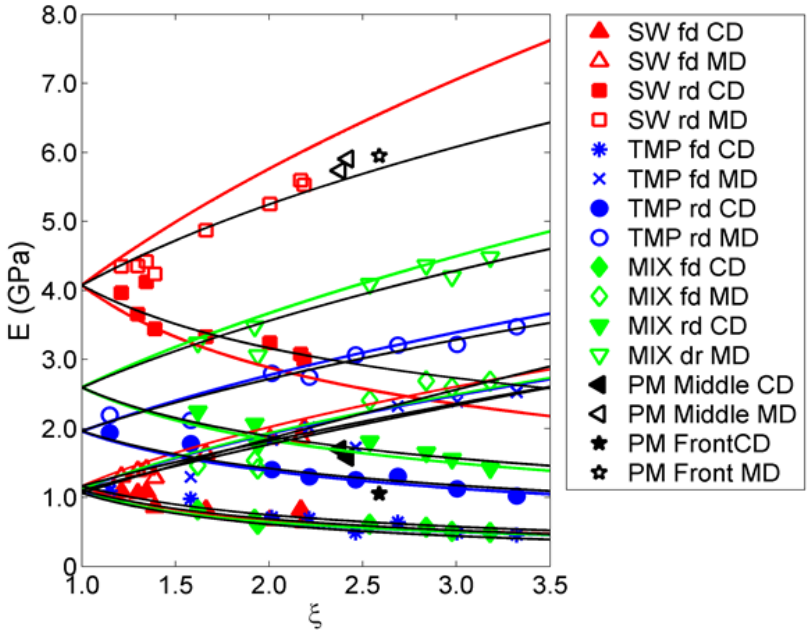

Fig 3 - Measured elastic modulus $E$ as a function of fiber orientation anisotropy $\xi$. The colored lines are relationships predicted by applying $E q 7$ and 8 . The black lines are fittings of Eq 10 as converted according to Eq 11 .

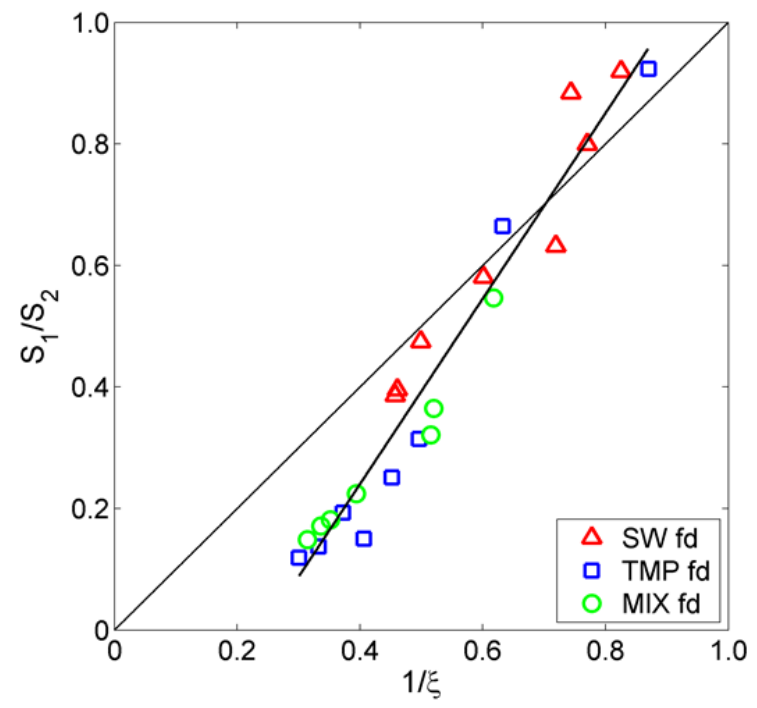

Fig 5 - Drying shrinkage anisotropy vs. the inverse of fiber orientation anisotropy. The diagonal line is based on Eq 12.

\section{Drying shrinkage anisotropy}

Wahlström (2004) and Wahlström and Mäkelä (2005) proposed the following relationship between drying shrinkage anisotropy and fiber orientation anisotropy:

$$
S_{1} / S_{2}=1 / \xi
$$

where $S$ is the drying shrinkage and subscript indices 1 and 2 refer to $\mathrm{MD}$ and $\mathrm{CD}$, respectively. According to the results of this study, presented in Fig $5,1 / \xi$ is not directly suitable for predicting the drying shrinkage anisotropy. The linear fitting yields $S_{1} / S_{2}=1.53 / \xi-$ 0.37 , and $r^{2}=0.948$. Certain factors, such as pressing conditions or the method for measuring fiber orientation, may have caused the deviation from earlier observations.

\section{Relationship between drying shrinkage and hygroexpansivity}

In his studies, Nordman (1958) found a linear relationship between drying shrinkage and hygroexpansivity; and that this is independent of pulp, beating or degree of restraining during drying. Similar

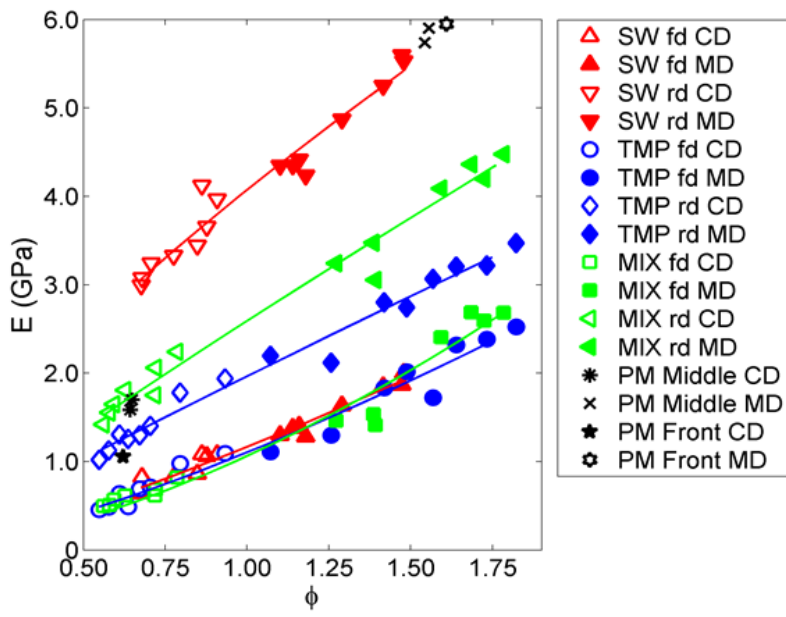

Fig 4 - The elastic modulus $E$ as a function anisotropy index $\phi$. The colored lines are fittings according to Eq 10.

correlations have also been found in other studies (Fahey, Chilson 1963; Manninen et al. 2011). Nordman stated, however, that in simulations of machine direction conditions by the uniaxial stretching of strips, the linear relationship no longer holds. Freely dried unbeaten pulp samples, having very low drying shrinkages $(S<3 \%)$, also fell from the linear fit. Salmén et al. (1987b) found that the changes in the degree of wet pressing have an effect on the relationship. Nanri and Uesaka (1993) also showed that the correlation between the drying shrinkage and hygroexpansion coefficient is not particularly high $\left(r^{2}=0.618\right)$ for freely-dried laboratory sheets of different pulps. A better correlation was achieved by comparing the drying shrinkage with the difference in hygroexpansion coefficients between freely dried and restraint-dried sheets.

A good correlation between the drying shrinkage and hygroexpansion coefficient of the freely dried samples can be observed from the results of this study in Fig 6 . The relationship is quite linear over the range of anisotropies measured, but between the different pulps, some slight differences in the slopes are detected. The fitting constants and coefficient of determination $\left(r^{2}\right)$ values are presented in Table 4 . The results presented in Nanri and Uesaka's (1993) study are plotted in red in Fig 7 and labeled "Reference". Although in principle the data points of Reference follow the trend of the results of this study, the scattering of different pulp samples clearly deviates from the slope of fitted line. The correlations of the results of this study do not improve if the difference in the hygroexpansion coefficients between freely dried and restraint-dried sheets is used instead, as can be seen from Fig 8 and from the $r^{2}$ values in Table 4.

These results indicate, at least for some limited cases, that both the hygroexpansion coefficient of freely dried sheets and the difference of the hygroexpansion coefficients have a linear dependence on drying shrinkage, and that this relationship is valid for samples having different fiber orientation anisotropies. If this result is combined with Nordman's (1958) observation that the linear relationship is also valid for samples 


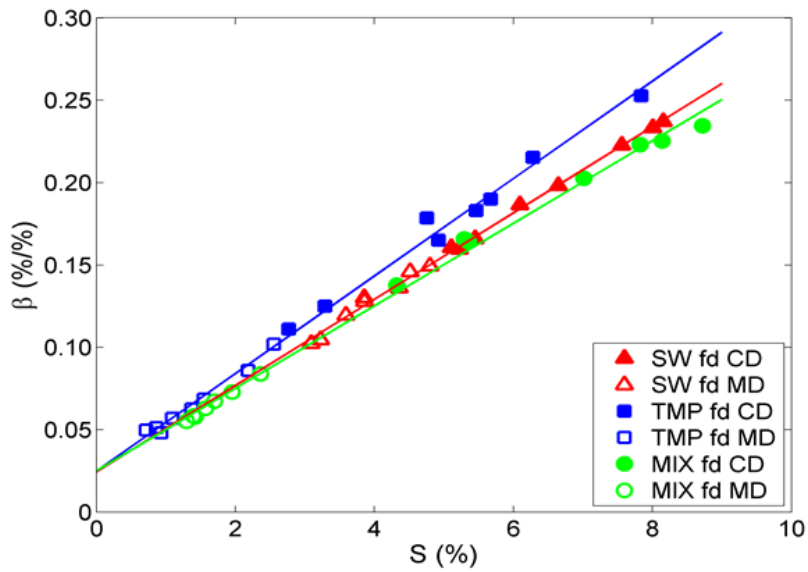

Fig 6 - Relationship between the drying shrinkage $S$ and the hygroexpansion coefficient $\beta$.

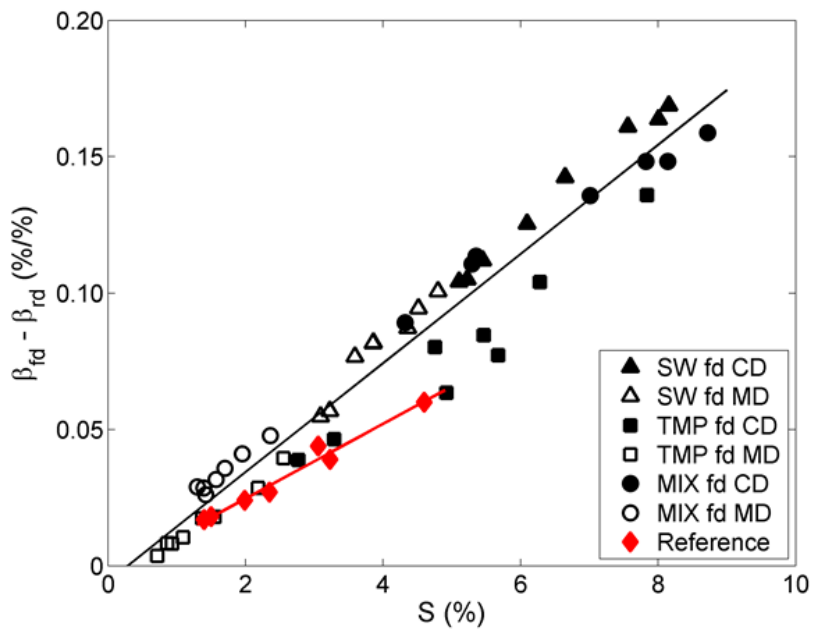

Fig 8 - Difference between freely dried and restraint-dried samples in the moisture expansion coefficient $\beta$ vs. the drying shrinkage $S$. Results of this study are in black; the reference results from Nanri and Uesaka's (1993) study are in red.

having different degrees of restraining during drying, it leads to an assumption that if an appropriate degree of restraining (or stretching) during drying results in a situation where no shrinkage has occurred $(S=0)$, the hygroexpansion coefficient of all samples would be independent of the anisotropy and measuring direction (MD or $\mathrm{CD}$ ). Then this zero shrinkage hygroexpansion coefficient should be the crossing point of the y-axis of the fittings of Fig 6, which is approximately $b=\beta_{0} \approx$ $0.025 \% / \%$ for all the pulps studied. To take the assumption even further, the fittings of Fig 6 predict that the hygroexpansion coefficient may be zero if the permanent strain of the sheet, due to the appropriate degree of stretching during drying, is roughly $b / a \approx 1 \%$ ( $a$ and $b$ are the fitting constants from Table 4).

The measured hygroexpansion coefficients are used to predict the drying shrinkage $\left(S_{\text {est }}\right)$ of restraint-dried laboratory sheets and production machine samples according to the assumption depicted above. The resulting estimates are presented in Fig 9 as a function of fiber orientation anisotropy. The estimated MD values of MIX samples with high fiber orientation anisotropy

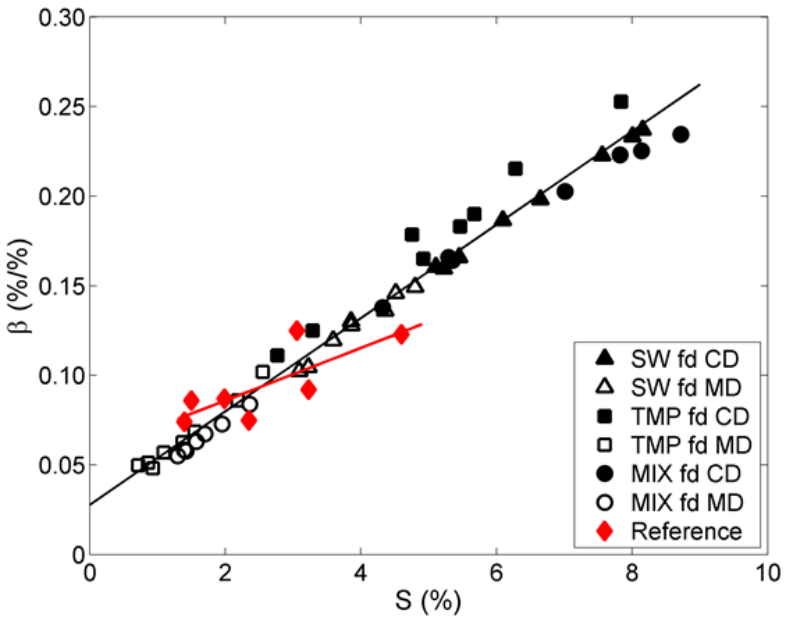

Fig 7 - Results of this study from Fig 6 are indicated by black markers. Reference results from Nanri and Uesaka's (1993) study are plotted in red.

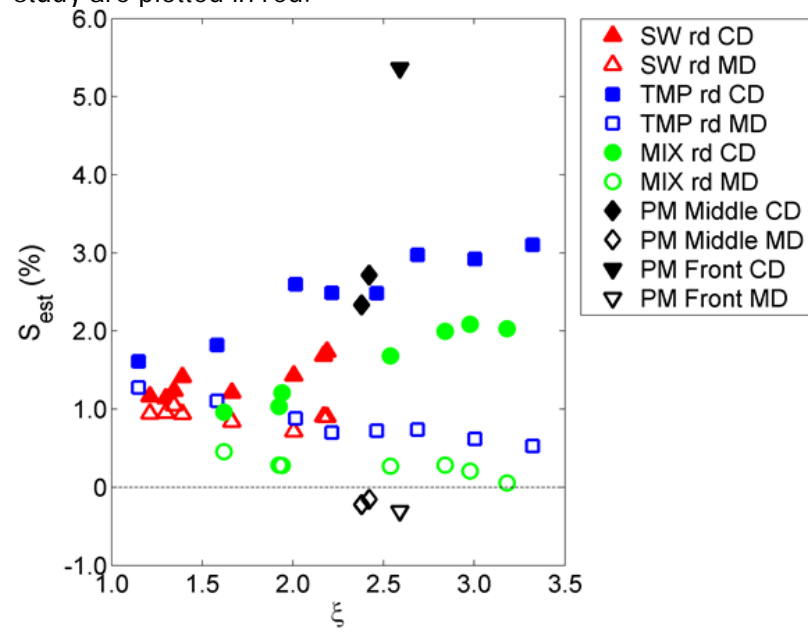

Fig 9 - Estimated shrinkage $S_{\text {est }}$ as a function of fiber orientation anisotropy.

Table 4 - The fitted constants $\left(\beta=a S+b\right.$ or $\beta_{f d}-\beta_{r d}=$ $a S+b)$ related to $F g$ 6-8 and the coefficient of determination.

\begin{tabular}{llll}
\hline Fig, samples & $\boldsymbol{a}(\mathbf{1} / \%)$ & $\boldsymbol{b}(\% / \%)$ & $\boldsymbol{r}^{\mathbf{2}}$ \\
\hline 6, SW & 0.026 & 0.024 & 0.997 \\
6, TMP & 0.030 & 0.025 & 0.995 \\
6, MIX & 0.025 & 0.025 & 0.996 \\
7, SW+TMP+MIX & 0.026 & 0.028 & 0.979 \\
7, Reference & 0.015 & 0.057 & 0.618 \\
8, SW+TMP+MIX & 0.020 & -0.006 & 0.947 \\
8, Reference & 0.014 & -0.003 & 0.976 \\
\hline
\end{tabular}

approach zero percent shrinkage $\left(\beta \approx \beta_{0}\right)$. For the TMP sheets, both the MD and CD values are higher, even rising to an estimated drying shrinkage of $3 \%$ in the case of the highly anisotropic CD samples. This high level of shrinkage seems unrealistic, although a certain amount of stress build-up during restraint drying (Wahlström, Fellers (1999)) might not have been relaxed before release, and thus the strain recovery may have decreased the dimensions of these sheets. Delayed strain recovery has also been detected by Skowronski and Robertson 
(1986). By examining the results of Nordman (1958), Nanko and $\mathrm{Wu}$ (1995) and Larsson and Wågberg (2008), it can be estimated that drying shrinkage of a totally restrained isotropic sheet may be on the order of $0.5 \%$. The estimated MD shrinkage $S_{\text {est }}$ of the production machine samples is negative for all positions (Middle: $0.15 \%$ and $-0.22 \%$, FS: $-0.31 \%$ ), indicating a slight elongation in the MD. The estimated CD shrinkage $S_{\text {est }}$ of the PM samples for the middle is $2.72 \%$ and $2.33 \%$, and for the front-side, $5.36 \%$. Based on wire marking frequency detection, the shrinkage difference between the middle and front-side samples was about 5.5\%. However, an accurate shrinkage value of the edge-position sample is difficult to determine, since the shrinkage difference between the left and right edges of the front side A4 sample was high, about $4 \%$. The MD and CD results of the middle samples seem reasonable, but the estimated CD shrinkage of the front-side sample is probably lower than the correct value. In uniaxial stretching, the detectable shrinkage includes not only hygroscopic shrinkage but also narrowing due to the MD stretching, which may be one reason why the assumption is not valid for the production machine samples.

\section{The relationship between the elastic modulus and hygroexpansivity}

The hygroexpansivity of an anisotropic sheet has been previously presented as a function of tensile stiffness or elastic modulus anisotropy (Uesaka 1994; Lyne et al. 1996). The hygroexpansion coefficient for equally wetpressed and dried sheets is studied herein as a function of the elastic modulus, as introduced in Fig 10. The simple power law fittings are applied separately for the different pulps:

$$
\beta=k_{2} E^{p}
$$

where $k_{2}$ and $p$ are the fitting parameters presented in Table 5.

The unique fitting can be extended to both freely and restraint-dried samples of same pulp. Even the production machine samples fit quite well in the MIX pulp curve, with the exception of $\mathrm{CD}$ results of the front-side sample. In that case, the elastic modulus, the hygroexpansivity or both are higher than expected, according to the laboratory sheet measurements. Eq 13 can be used to determine individual equations for $\mathrm{MD}$ and $\mathrm{CD}$ as a function of the geometric mean and elastic modulus anisotropy

$$
\beta=k_{2} E_{\text {geom }}^{p}\left(E_{1} / E_{2}\right)^{p \times s} \text {. }
$$

The constant $k_{2} E_{\text {geom }}^{p}$, power $p \times s$ and coefficient of determination $\left(r^{2}\right)$ are listed in Table 6. The fittings of $E q 13$ with the change of variables according to Eq 14 are presented in Fig 11 as colored lines. The direct fittings are made using power equation

$$
\beta=k_{3}\left(E_{1} / E_{2}\right)^{t} \text {. }
$$

Fitting parameters $k_{3}$ and $t$ are presented in Table 7, and the fitted curves are plotted as black lines in Fig 11. The poor $r^{2}$ of the fittings of the SW-rd-MD samples are also an indication of the almost nonexistent anisotropy dependency not being able to exceed the measuring inaccuracy. In that case, the average of the hygroexpansion values of the SW-rd-MD samples provides an equally good estimate as the power law fitting. Accordingly, Uesaka (1994) concluded that particularly for restraint-dried, low-density sheets, the MD hygroexpansivity does not show significant dependence on the elastic stiffness ratio.

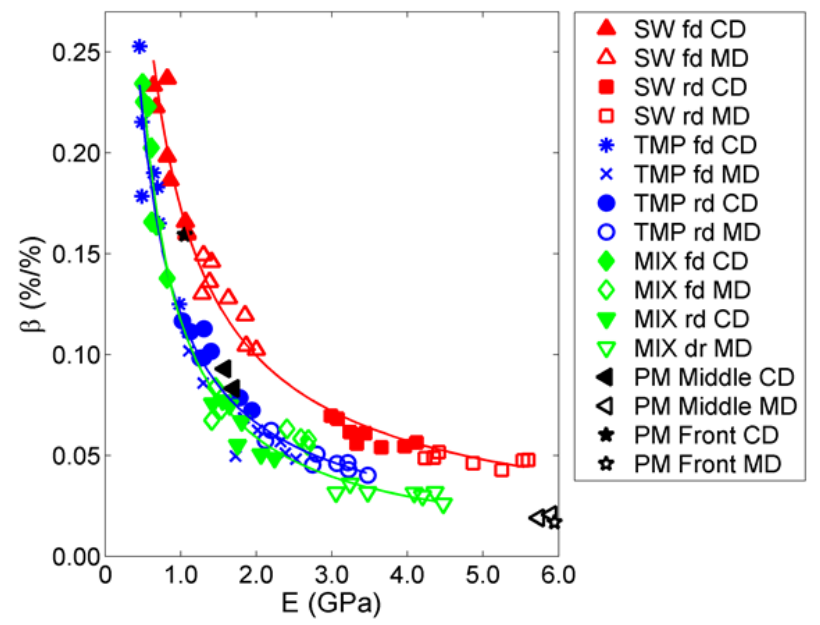

Fig 10 - Moisture expansion coefficient $\beta$ as a function of the elastic modulus $E$ and fitted curves ( $E q 13$ ) for different pulp types.

Table 5 - Fitted parameters of Eq 13.

\begin{tabular}{llll}
\hline Pulp & $\boldsymbol{k}_{\mathbf{2}}\left((\% / \%) \mathrm{GPa}^{-\boldsymbol{p}}\right)$ & $\boldsymbol{p}$ & $\boldsymbol{r}^{\mathbf{2}}$ \\
\hline SW & 0.172 & -0.795 & 0.977 \\
TMP & 0.120 & -0.854 & 0.956 \\
MIX & 0.117 & -0.987 & 0.979 \\
\hline
\end{tabular}

Table 6 - Fitted parameters of Eq 14. $p \times s$ gets negative values for $M D$ and positive for $C D$.

\begin{tabular}{lllll}
\hline $\begin{array}{l}\text { Pulp and } \\
\text { drying }\end{array}$ & $\begin{array}{l}\boldsymbol{k}_{\mathbf{2}} \boldsymbol{E}_{\boldsymbol{g e o m}}^{\boldsymbol{p}} \\
(\% / \%)\end{array}$ & $|\boldsymbol{p} \times \boldsymbol{s}|$ & $\begin{array}{l}\text { MD } \\
\boldsymbol{r}^{\mathbf{2}}\end{array}$ & $\begin{array}{l}\text { CD } \\
\boldsymbol{r}^{\mathbf{2}}\end{array}$ \\
\hline SW fd & 0.153 & 0.397 & 0.703 & 0.876 \\
SW rd & 0.056 & 0.397 & -1.405 & 0.150 \\
TMP fd & 0.110 & 0.427 & 0.789 & 0.945 \\
TMP rd & 0.067 & 0.427 & 0.895 & 0.752 \\
MIX fd & 0.105 & 0.493 & 0.301 & 0.969 \\
MIX rd & 0.046 & 0.493 & 0.190 & 0.827 \\
\hline
\end{tabular}

Table 7 - Fitted parameters of Eq 15.

\begin{tabular}{llll}
\hline $\begin{array}{l}\text { Pulp, drying and } \\
\text { direction }\end{array}$ & $\boldsymbol{k}_{\mathbf{3}}(\% / \%)$ & $\boldsymbol{t}$ & $\boldsymbol{r}^{\mathbf{2}}$ \\
\hline SW fd MD & 0.155 & -0.344 & 0.821 \\
SW fd CD & 0.152 & 0.408 & 0.877 \\
SW rd MD & 0.050 & -0.153 & 0.385 \\
SW rd CD & 0.053 & 0.394 & 0.734 \\
TMP fd MD & 0.101 & -0.452 & 0.969 \\
TMP fd CD & 0.107 & 0.464 & 0.959 \\
TMP rd MD & 0.063 & -0.371 & 0.951 \\
TMP rd CD & 0.073 & 0.407 & 0.912 \\
MIX fd MD & 0.096 & -0.321 & 0.904 \\
MIX fd CD & 0.113 & 0.433 & 0.989 \\
MIX rd MD & 0.038 & -0.269 & 0.688 \\
MIX rd CD & 0.038 & 0.648 & 0.933 \\
\hline
\end{tabular}



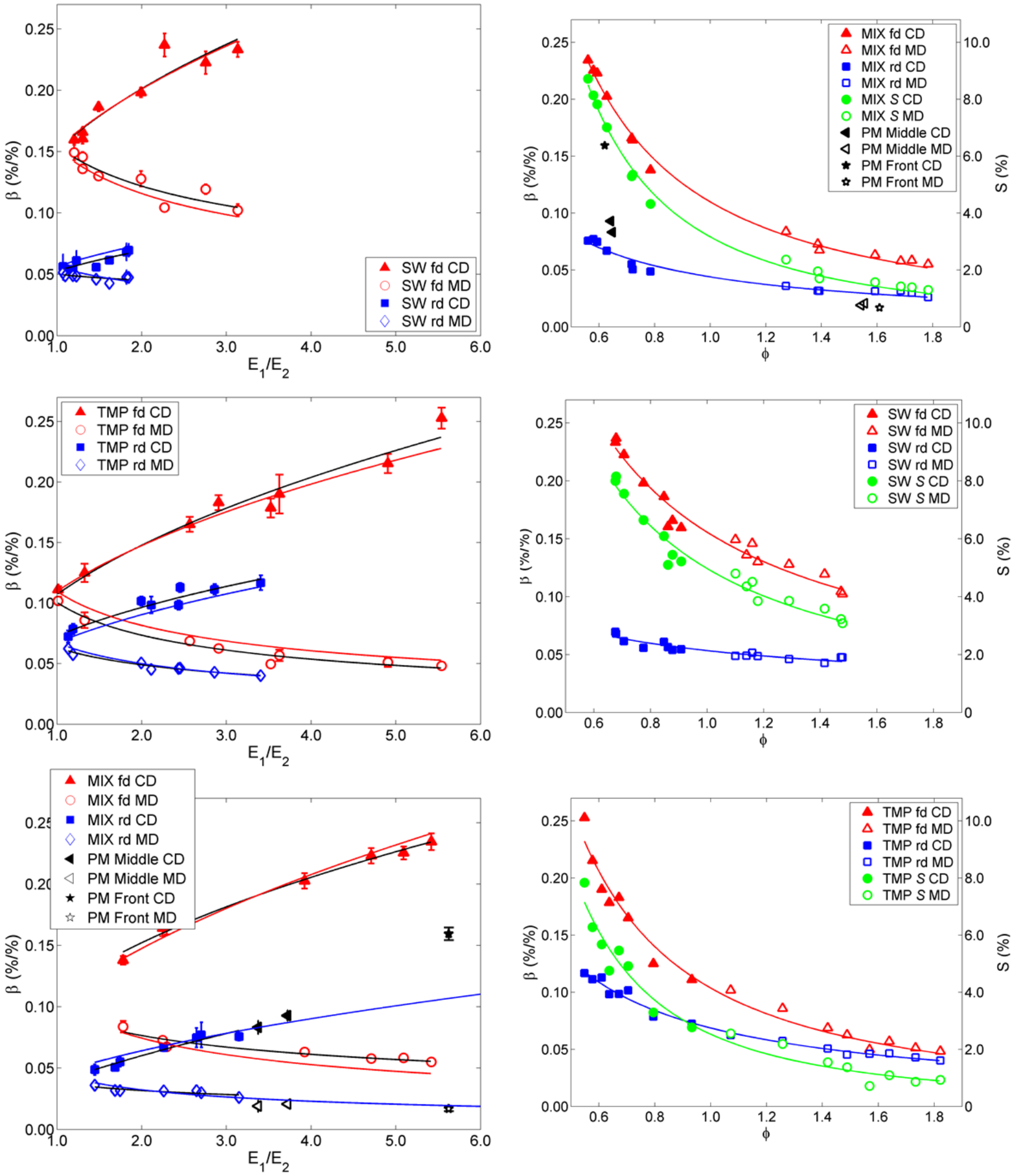

Fig 11 - Hygroexpansion $\beta$ as a function of the elastic modulus anisotropy $E_{1} / E_{2}$. The fittings of $E q 13$ converted according to $E q 14$ are depicted by colored lines, and the fittings of $E q 15$ by black lines. The error bars indicate the $95 \%$ confidence intervals.

\section{The relationship of hygroexpansivity and drying shrinkage to fiber orientation}

The fiber orientation anisotropy $\xi$ can be transformed into a directional parameter by using the anisotropy index $\phi$. The hygroexpansion coefficient $\beta$ and drying shrinkage $S$ as a function of $\phi$, is presented in Fig 12. The parameters $k_{4}$ and $v$ of the power fittings

$$
\beta=k_{4} \phi^{v} \text { or } S=k_{4} \phi^{v}
$$

Fig 12 - Fitted curves ( $E q$ 16) for hygroexpansion $\beta$ and drying shrinkage $S$ as a function of the anisotropy index $\phi$.

and the coefficient of determination $r^{2}$ are presented in Table 8.

Both the MD and $\mathrm{CD}$ of one pulp treated under an equivalent degree of restraint during drying are fitted in a single power curve. The restraining decreases the absolute value of power $v$ and factor $k_{4}$, indicating that both the hygroexpansivity level and the variability due to the anisotropy and the measuring direction decrease when drying shrinkage is prevented. If the assumption of the 
linear relationship would hold, the appropriate amount of stretching during drying (given zero drying shrinkage) would result in a power equal to 0 , and dependency of hygroexpansivity on anisotropy would disappear. However, in every case the drying shrinkage has slightly higher powers than that of hygroexpansion coefficient of freely dried sheets, indicating that the relationship between hygroexpansivity and drying shrinkage is not strictly linear.

The change of the variable from the anisotropy index to fiber orientation anisotropy can be performed using Eq 17:

$$
\beta=k_{4} \xi^{v \times s} \text {. }
$$

The results are presented in Fig 13. The coefficient of determination $r^{2}$ values calculated individually for samples SW-rd-MD and MIX-rd-MD (Table 9) are poor, but low values for those samples are also observed when direct power law fittings (Table 10) are employed. This could be indication of the low dependency of hygroexpansion on anisotropy as well as an indication of poor fitting quality. Although SW pulp has both the highest shrinkage potential and the steepest slope of anisotropy dependency of the freely dried samples, it also has the lowest hygroexpansivity when SW restraint-dried samples are compared with TMP and MIX samples. In accordance with this phenomenon, Nanri and Uesaka (1993) found indications that the effect of drying restraint on hygroexpansivity for chemical pulps is higher than that for mechanical pulps, because of the higher drying shrinkage potential of the chemical pulps. The restraintdried SW samples show only a weak dependence on anisotropy when compared to the TMP and MIX pulps. Restrain or stretching narrows the hygroexpansivity differences between different pulps or other treatments, as has been observed in several studies (Fahey, Chilson 1963; Nordman 1958; Stamm, Beasley 1961; Salmén et al. 1987b).

Stretching during drying decreases the MD hygroexpansivity of the production machine samples under the curve of the restraint-dried laboratory samples. The CD hygroexpansivity of the production machine samples falls between the restraint-dried and freely dried laboratory sheet results.

Table 8 - Fittd parameters of Eq 16.

\begin{tabular}{llll}
\hline Pulp and drying & \multicolumn{1}{c}{$\boldsymbol{k}_{\mathbf{4}}$} & $\boldsymbol{v}$ & $\boldsymbol{r}^{\mathbf{2}}$ \\
\hline SW fd $\beta$ & $0.156(\% / \%)$ & -0.981 & 0.961 \\
SW rd $\beta$ & $0.053(\% / \%)$ & -0.499 & 0.863 \\
SW fd $S$ & $4.973(\%)$ & -1.164 & 0.965 \\
TMP fd $\beta$ & $0.104(\% / \%)$ & -1.343 & 0.982 \\
TMP rd $\beta$ & $0.069(\% / \%)$ & -0.900 & 0.986 \\
TMP fd $S$ & $2.527(\%)$ & -1.734 & 0.970 \\
MIX fd $\beta$ & $0.110(\% / \%)$ & -1.302 & 0.995 \\
MIX rd $\beta$ & $0.044(\% / \%)$ & -0.901 & 0.956 \\
MIX fd $S$ & $3.164(\%)$ & -1.710 & 0.995 \\
\hline
\end{tabular}
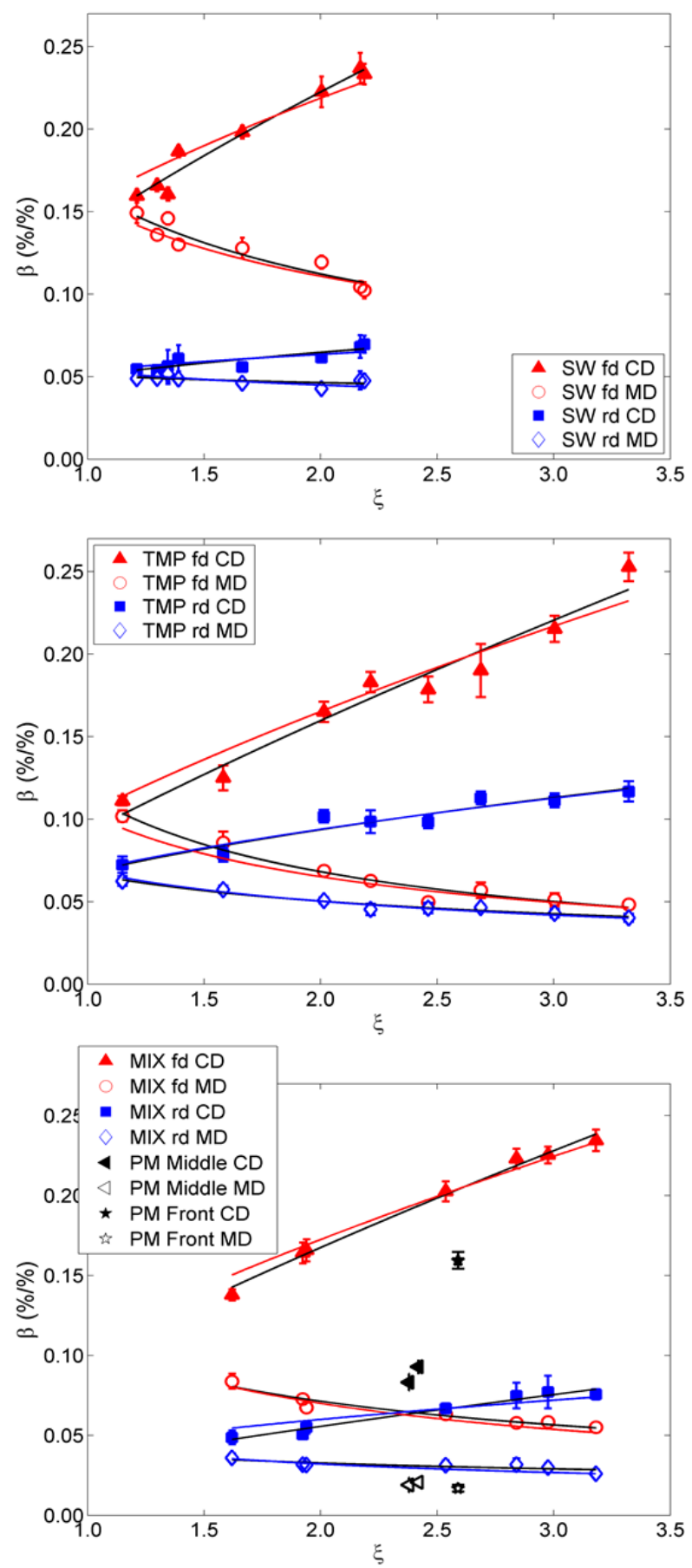

Fig 13 - Hygroexpansion coefficient $\beta$ as a function of fiber orientation anisotropy $\xi$. The fittings of $E q 16$ converted according to Eq 17 are shown with colored lines, and the direct power law fittings with the black lines.

Table 9 - Fitted parameters of Eq 17. $v \times s$ gets negative values for $M D$ and positive for $C D$.

\begin{tabular}{lllll}
\hline $\begin{array}{l}\text { Pulp, drying } \\
\text { and direction }\end{array}$ & $\begin{array}{l}\boldsymbol{k}_{\mathbf{4}} \\
(\% / \%)\end{array}$ & $|\boldsymbol{v} \times \boldsymbol{s}|$ & $\begin{array}{l}\text { MD } \\
\boldsymbol{r}^{\mathbf{2}}\end{array}$ & $\begin{array}{l}\text { CD } \\
\boldsymbol{r}^{\mathbf{2}}\end{array}$ \\
\hline SW fd & 0.156 & 0.490 & 0.847 & 0.895 \\
SW rd & 0.053 & 0.249 & 0.074 & 0.679 \\
TMP fd & 0.104 & 0.672 & 0.910 & 0.933 \\
TMP rd & 0.069 & 0.450 & 0.947 & 0.917 \\
MIX fd & 0.110 & 0.651 & 0.881 & 0.972 \\
MIX rd & 0.044 & 0.451 & 0.300 & 0.813 \\
\hline
\end{tabular}


Table 10 - Direct power law fittings $\left(\beta=k_{5} \phi^{w}\right)$ presented in Fig 13.

\begin{tabular}{llll}
\hline $\begin{array}{l}\text { Pulp, drying and } \\
\text { direction }\end{array}$ & $\boldsymbol{k}_{\mathbf{5}}(\% / \%)$ & \multicolumn{1}{l}{$\boldsymbol{w}$} & $\boldsymbol{r}^{\mathbf{2}}$ \\
\hline SW fd MD & 0.163 & -0.542 & 0.895 \\
SW fd CD & 0.141 & 0.663 & 0.965 \\
SW rd MD & 0.051 & -0.132 & 0.353 \\
SW rd CD & 0.050 & 0.362 & 0.752 \\
TMP fd MD & 0.115 & -0.755 & 0.959 \\
TMP fd CD & 0.092 & 0.795 & 0.952 \\
\hline
\end{tabular}

\begin{tabular}{llll}
\hline $\begin{array}{l}\text { Pulp, drying and } \\
\text { direction }\end{array}$ & $\boldsymbol{k}_{\mathbf{5}}(\% / \%)$ & $\boldsymbol{w}$ & $\boldsymbol{r}^{\mathbf{2}}$ \\
\hline TMP rd MD & 0.067 & -0.411 & 0.956 \\
TMP rd CD & 0.068 & 0.465 & 0.918 \\
MIX fd MD & 0.106 & -0.573 & 0.932 \\
MIX fd CD & 0.099 & 0.761 & 0.992 \\
MIX rd MD & 0.040 & -0.293 & 0.656 \\
MIX rd CD & 0.033 & 0.754 & 0.963 \\
\hline
\end{tabular}

\section{Conclusions}

A considerably linear relationship between the hygroexpansion coefficient of freely dried sheets and drying shrinkage from press-dry to equilibrium conditions was observed for laboratory sheets having different fiber orientation anisotropies. The corresponding linear relationship had earlier been observed to be valid for equally pressed samples dried freely or under different degree of restraining (Nordman 1958). In this study, some deviations were noted to arise due to the different pulp or in cross directional values of cross profile edge samples produced under uniaxial draw of the production machine. Based on the linearity of relationship, the following assumption was made: an adequate degree of restraint may remove the anisotropy dependency of the hygroexpansion coefficient. However, in reality, neither the linearity is complete nor the degree of restraint (or stretching) could be arranged in such a way that would subsequently lead to a situation in which the permanent strain is zero for all directions of an anisotropic sheet. Nevertheless, a very low dependency of the hygroexpansion coefficient on anisotropy was achieved with the restraint-dried samples, providing a way to minimize problems coming from local or twosided variation of hygroexpansivity, for example. Condebelt drying is an excellent example of the effect of restraint drying on improving dimensional stability (Retulainen, Hämäläinen 2000) and suppressing cockling tendency. Irreversible hygroexpansion behavior and recoverable strain due to humidification may, however, change the hygroexpansion behavior, but those phenomena did not fall within the scope of this research.

It is interesting to note that independently both the measurement direction (MD and $\mathrm{CD}$ ) and drying option (freely and restraint) all hygroexpansion coefficient values of a particular pulp fall on a single power curve when studied as a function of elastic modulus. This relationship does not necessarily imply causation, but both properties, hygroexpansion coefficient and elastic modulus, depend on anisotropy and drying restraint. Using the anisotropy index, the fiber orientation anisotropy was converted into a directional variable. When anisotropy index is used, the MD and CD hygroexpansion coefficient may also be fitted on a single curve, while the freely and restraint-dried sheets evidently require two different fitting curves. An equivalent power law fitting can also be used when the relationship between the drying shrinkage and the anisotropy index are studied. These fittings can be restored as a function of the anisotropy of the elastic

modulus or the anisotropy of fiber orientation with a simple change of the variable.

The fittings of the hygroexpansion coefficient as a function of the anisotropy index and the relationship between drying shrinkage and hygroexpansivity will be used in construction of a hygroexpansivity-shrinkage model. This hygroexpansivity-shrinkage model together with an elasto-plastic material model based on the experimental stress-strain curve parameters presented in Erkkilä et al. (2013), have been incorporated as a part of the continuum mechanical modeling approach presented in Lipponen et al. (2009) and Erkkilä et al. (2015).

\section{Acknowledgements}

The authors are thankful to Collin Hii and Juha Happonen for their high contribution on the experimental results.

\section{Literature \\ de Ruvo, A., Lundberg, S., Martin-Löf, S., Södermark, C. (1976): Influence of temperature and humidity on the elastic and expansional properties of paper and the constituent fibre, In: The Fundamental Properties of Paper Related to Its Uses, Transactions of the Symposium 1973, F. Bolan (ed.) Brittish Paper Board Industry Federation, London, 785 -806.}

Erkkilä, A.-L., Pakarinen, P. and Odell, M. (1998): Sheet forming studies using layered orientation analysis, Pulp Paper Can. 99(1), 81-85.

Erkkilä A.-L., Leppänen T. and Hämäläinen J. (2013): Empirical plasticity models applied for paper sheets having different anisotropy and dry solids levels, International Journal of Solids and Structures 50(14-15), 2151-2179.

Erkkilä A.-L., Leppänen T., Hämäläinen J. and Tuovinen, T. (2015): Hygro-elasto-plastic model for planar orthotropic material, International Journal of Solids and Structures (Accepted 2015, doi:10.1016/j.ijsolstr.2015.02.001).

Fahey, D.J. and Chilson, W.A. (1963): Mechanical treatments for improving dimensional stability of paper, Tappi 46(7), 393399.

Hii, C. (2008): Hygroexpansivity of paper, M.Sc. Thesis, Helsinki University of Technology, Espoo, Finland.

Htun, M. and Fellers, C. (1982): The invariant mechanical properties of oriented handsheets, Tappi 65(4), 113-117.

Larsson P.A. and Wågberg L. (2008): Influence of fibre-fibre joint properties on the dimensional stability of paper, Cellulose 15(4), 515-525.

Li, Z., Li, K., Camm, C. and Chen, Z. (2009): Dimensional stability of paper made from Mixtures of BCTMP and Kraft Fibres, J. Pulp Paper Sci. 35(3-4), 123-129. 
Lipponen, P., Erkkilä, A.-L., Leppänen, T. and Hämäläinen, J. (2009): On the importance of in-plane shrinkage and throughthickness moisture gradient during drying on cockling and curling phenomena, In: I'Anson S.J. (ed.), Advances in Pulp and Paper Science and Technology, The Pulp and Paper Fundamental Research Society, Manchester, pp. 389-436.

Lyne, Å., Fellers C. and Kolseth, P. (1996): The effect of filler on hygroexpansivity, Nord. Pulp Paper Res. J. 11(3), 152-163.

Manninen, M., Kajanto, I., Happonen, J. and Paltakari, J. (2011): The effect of microfibrillated cellulose addition on drying shrinkage and dimensional stability of wood-free paper, Nord. Pulp Paper Res. J. 26(3), 297-305.

Nanko, H. and Wu, J. (1995): Mechanisms of paper shrinkage during drying, Int. Paper Physics Conf., Niagara-on-the-lake, Ontario, Canada, September 11-14, 1995, C.P.P.A and Tappi, pp. 103-113.

Nanri, Y. and Uesaka, T. (1993): Dimensional stability of mechanical pulps - drying shrinkage and hygroexpansivity, Tappi J. 76(6), 62-66.

Nordman, L.S. (1958): Laboratory investigations into the dimensional stability of paper, Tappi 41(1), 23-50.

Norman, B. (1989): Overview of the physics of forming, In: Baker, C.F. and Punton, V.W. (eds.), Fundamentals of papermaking, Vol. 3, Mechanical Engineering Publications London, pp. 674-693.

Page D.H. and Tydeman P.A. (1962): A new theory of the shrinkage, structure and properties of paper, In: Bolam, F. (ed.), The formation and structure of paper, Vol. 2, British Paper and Board Makers' Assoc. London, pp. 397-413.

Rance, H.F. (1954): Effect of water removal on sheet properties, Tappi 37(12), 640-648.

Retulainen, E. and Hämäläinen, A. (2000): Three years of Condebelt drying at Stora Enso's Pankakoski mill, Tappi J. 83(5), 84, Tappi web site 1-8.

Salmén, L., Fellers, C. and Htun, M. (1987a): The development and release of dried-in stresses in paper, Nord. Pulp Paper Res. J. 2(2), 44-48.
Salmén, L., Boman, R., Fellers, C. and Htun, M. (1987b): The implications of fiber and sheet structure for the hygroexpansivity of paper, Nord. Pulp Paper Res. J. 2(4), 127-131.

Schrier, B.H. and Verseput, H.W. (1967): Evaluating the performance of folding cartons, Tappi 50(3), 114-117.

Skowronski, J. and Robertson, A.A. (1986): The deformation properties of paper: tensile strain and recovery, J. Pulp Paper Sci. 12(1), 20-25

Stamm, A.J. and Beasley, J.N. (1961): Dimensional stabilization of paper by acetylation, Tappi 44(4), 271-275.

Uesaka, T., Moss, C. and Nanri, Y. (1992): The characterisation of hygroexpansivity of paper, J. Pulp Paper Sci. 18(1), 11-16.

Uesaka, T. (1994): General formula for hygroexpansion of paper, Journal of Material Science 29(9), 2373-2377.

Uesaka, T. and Qi, D. (1994): Hygroexpansivity of paper Effects of fibre-to-fibre bonding, J. Pulp Paper Sci. 20(6), 175179.

Wahlström, T. and Fellers, C. (1999): Biaxial straining of paper during drying, relations between stresses, strains and properties, In: Tappi Engineering Conference, Anaheim, CA, USA, September 12-16, 1999, pp. 705-720.

Wahlström, T. (2004): The invariant shrinkage and stiffness of paper - modeling anisotropic behaviour based on isotropic handsheets, In: Progress in Paper Physics Seminar, Trondheim, Norway, June 21-24, 2004, pp. 105.

Wahlström, T. and Mäkelä, P. (2005): Predictions of anisotropic multiply board properties based on isotropic ply properties and drying restraints, In: I'Anson S.J. (ed.), Advances in Paper Science and Technology, The Pulp and Paper Fundamental Research Society, Manchester, pp. 241281.

Manuscript received September 18, 2014 Accepted March 3, 2015 